\title{
THE DESIGN AND PHYSICS PERFORMANCE OF THE ITER UPPER PORT ECH FRONT STEERING LAUNCHER
}

\author{
M.Henderson ${ }^{\mathrm{a}}$, R. Chavan ${ }^{\mathrm{a}}$, R. Heidinger ${ }^{\mathrm{b}}$, G. Ramponi ${ }^{\mathrm{c}}$, G. Saibene ${ }^{\mathrm{d}}$, F. Sanchez ${ }^{\mathrm{a}}$, O. Sauter ${ }^{\mathrm{a}}$, H. Shidara ${ }^{\mathrm{a}}$, and \\ H. Zohm ${ }^{\mathrm{e}}$ \\ ${ }^{a}$ Centre de Recherche en Physique des Plasmas - Ecole Polytechnique Federale de Lausanne,Lausanne, Switzerland \\ ${ }^{b}$ Forschungszentrum Karlsruhe, Karlsruhe, Germany \\ ${ }^{c}$ Istituto di Fisica del Plasma, Milano, Italy \\ ${ }^{d}$ EFDA Close Support Unit, Garching, Germany \\ e IPP-Garching, Max-Planck-Institut für Plasmaphysik, Garching, Germany
}

\begin{abstract}
The purpose of the ITER electron cyclotron resonance heating (ECRH) upper port launcher will be to stabilize the neoclassical tearing mode (NTM) by driving currents (ECCD) locally inside either the $q=3 / 2$ or 2 island. In order to deposit current predominately inside the island, a narrow ECCD current deposition profile is required. Also, a wide steering range is desired for application of co-ECCD on the relevant flux surfaces over the wide spectra of possible ITER plasma equilibria. A front steering (FS) launcher has been designed for application on the ITER upper port, offering a wider poloidal steering angle $\left(\geq \mathbf{2 0}{ }^{\circ}\right)$ and a higher ECCD density (factor of 3.0 on average) than the presently planned remote steering (RS) launcher. The launcher is capable of injecting $16 \mathrm{MW}$ per port (eight beams of $2.0 \mathrm{MW}$ ) using a two mirror system (1 focusing and 1 steering) for focusing and redirecting the beam towards either the $q=3 / 2$ or 2 flux surfaces for all envisioned plasma equilibria. The steering mechanism is bearing-free with flexure pivots, in a compact cartridge capable of $\pm 12^{\circ}$ rotation (corresponding to a poloidal steering range of $\pm 24^{\circ}$ for the microwave beam). The increased steering range enlarges the range in which the ECCD deposition can be applied, offering the potential to address other physics issues such as sawtooth, FIR or ELM control. The complete design concept and calculated ECCD performance for NTM stabilization will be presented
\end{abstract}

\section{Keywords-ITER; ECRH; Launcher; NTM stabilisation}

\section{INTRODUCTION}

The purpose of the ITER electron cyclotron resonance heating (ECRH) upper port antenna (or launcher) will be to drive current (ECCD) locally inside the island which forms on the $q=3 / 2$ or 2 rational magnetic flux surfaces in order to stabilize the neoclassical tearing mode (NTM). The launcher should be capable of steering the ECCD current deposition profile $\left(\mathrm{j}_{\mathrm{CD}}\right)$ across the resonance surface over the range in which the $q=3 / 2$ and 2 surfaces are found, for the various plasma equilibria susceptible to the onset of NTMs, as shown in figure 1 . Also, $\mathrm{j}_{\mathrm{CD}}$ must be narrow relative to the island width and its amplitude greater than that of the bootstrap current $\left(\mathrm{j}_{\mathrm{BS}}\right)$ found outside the island in order to effectively stabilize the NTM. The ratio of these two currents, $\max \left(\mathrm{j}_{\mathrm{CD}}\right) / \mathrm{j}_{\mathrm{BS}}$, provides an NTM stabilisation figure of merit $\left(\eta_{\text {NTM }}\right)$. The physics objective for the launcher is to achieve $\eta_{\mathrm{NTM}}>1.2$ for the various relevant plasma equilibria that are susceptible to NTMs (equilibria 2, 3a and 5) [1]. The European Fusion Development Agreement is currently in the process of developing two launcher designs: remote steering (RS) [2] and front steering (FS) launchers. Both launchers are capable of handling 24 beams (eight $1.0 \mathrm{MW}$ beams) but compatible with $2.0 \mathrm{MW}$ injection per beam. The two launcher designs are being designed in parallel, with the goal of providing the optimum launcher based on ITER's engineering and physics requirements. The RS launcher [3, 4] offers the advantage of not requiring moving parts within the vessel vacuum boundary (far from the thermal and nuclear radiation of the plasma). However, it has a limited angular range $(\leq$ $\pm 12^{\circ}$ ) and projects a relatively broad beam spot size $(>55 \mathrm{~mm})$ at the resonance surface and has only 6 beams/port (due to space limitations at the port entrance). By contrast, the FS launcher [5 - 7] offers an extended angular range ( up to $\pm 24^{\circ}$ ) and projects a much narrower spot size on the resonance surface, but requires a rotatable mirror near the plasma. The mm-wave components are more compact, fitting 8 beams per port requiring a total of only three ports relative to 4 ports for the RS launcher. A similar FS launcher is already planned for the equatorial port $[8,9]$.

The ECCD is the only current source that is both localized and steerable using external actuators and, therefore, should be used to its maximum capabilities on ITER. This implies the effective use of each MW injected, but also employing the ECCD in the widest possible range of physics applications. The FS launcher has been shown to be more effective than the RS launcher in stabilizing the NTM [10] with a factor of 3 increase in $\eta_{\mathrm{NTM}}$ over that achievable with the RS. In addition, the steering range of the FS launcher can be increased in order to expand the physics applications beyond NTM stabilization and address additional physics issues such as sawtooth control, ELM destabilization, Frequently Interrupted Regime (FIR) 
control, etc. After a brief description of the FS launcher, the NTM stabilization efficiency of the launcher will be described followed by the possible extension of the physics applications.

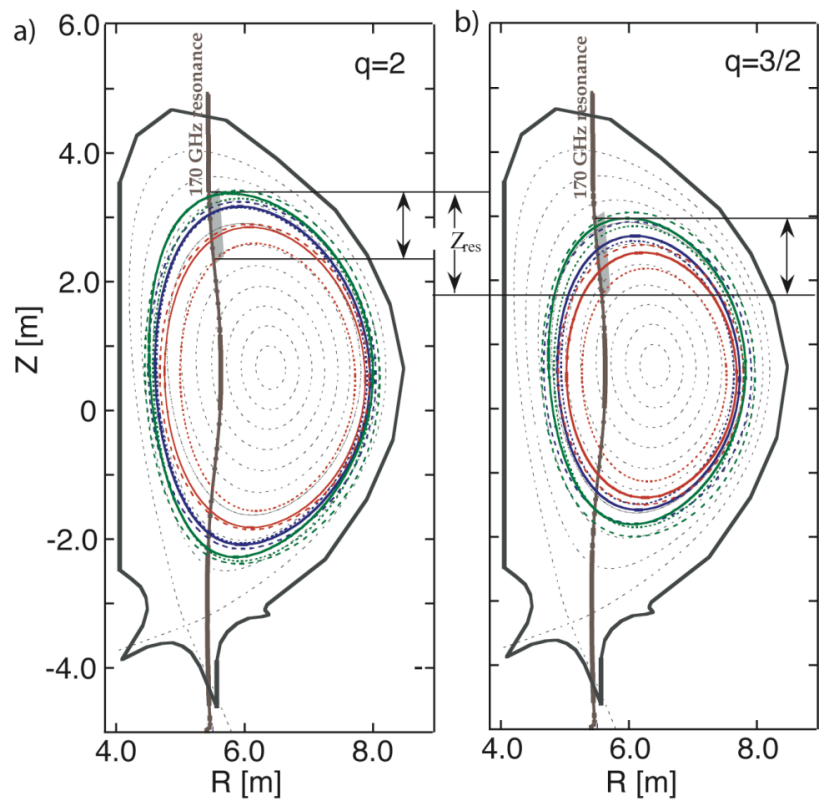

Figure 1. The (a) $q=2$ and (b) 3/2 flux surfaces susceptible to the NTM, with $\mathrm{Z}_{\text {res }}$ indicating the region that the launcher must access along the resonance surface. [7]

\section{FS LAUNCHER DESCRIPTION}

A simplified poloidal view of the present FS launcher design is shown in figure 2. Eight circular waveguides enter at the port entrance on the right, with the waveguides arranged in two rows of four. A miter bend 'dog-leg' assembly is used to direct the 8 beams (both in the toroidal and poloidal directions) to one single focusing mirror, with the incident beams partially overlapping in both directions. The reflected beams are then directed downward to two separate flat steering mirrors, which redirect the beams into the plasma with a toroidal injection angle of $\beta \sim 20^{\circ}$. The beams are allowed to expand from the waveguide aperture, so that they can be refocused to a narrow waist $(21.0 \mathrm{~mm})$ far into the plasma ( $>1.7 \mathrm{~m}$ after steering mirror). The angular rotation of the steering mirror provides access along the resonance layer from a height of 1.8 to $3.4 \mathrm{~m}$ (corresponding to a mirror angular rotation of $< \pm 6.0^{\circ}$ or $\pm 12.0^{\circ}$ for the beam), providing access to all relevant $\mathrm{q}=2$ and $3 / 2$ rational flux surfaces.

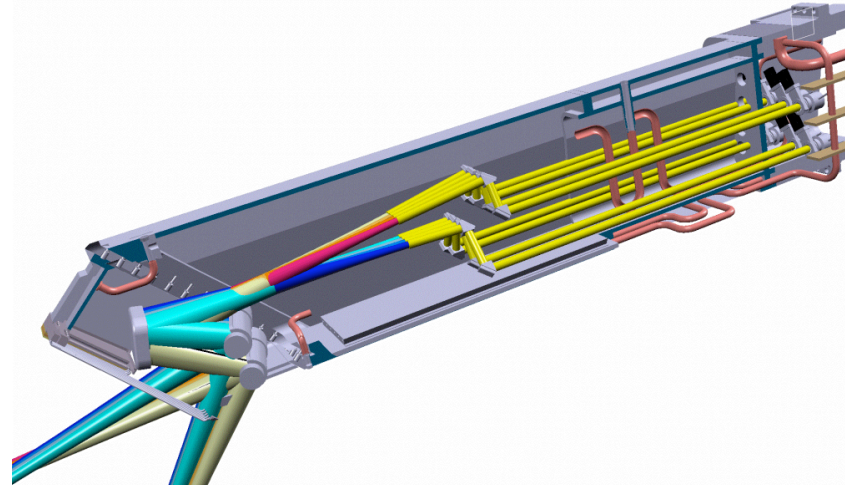

Figure 2. The FS launcher installed in the upper port plug. [7]

The steering mechanism [12] is the critical component of the FS design and a failure of one steering mechanism would render 4 RF beams unusable. The FS launchers on existing tokamaks have been crippled when the steering mechanism grips, which typically occurs between two moving surfaces. The steering mechanism proposed avoids all frictional surfaces. Traditional bearings are replaced with flexure pivots and the movement is controlled using a pneumatic (helium) system using bellows pushing against springs, see figure 3 . A coiled cooling tube with either a single or double wall is envisioned to provide a flexible coolant feed to the mirror, following a similar design to that proposed for the equatorial launcher $[8,9]$ but with reduced stresses.

It is important to note that a failed steering mechanism can only be replaced during a normal tokamak opening. However, four of the six steering mechanisms could fail and still provide an averaged performance equivalent to that of a fully operational 24-beam RS launcher ( 8 beam/3 port all purpose launcher).

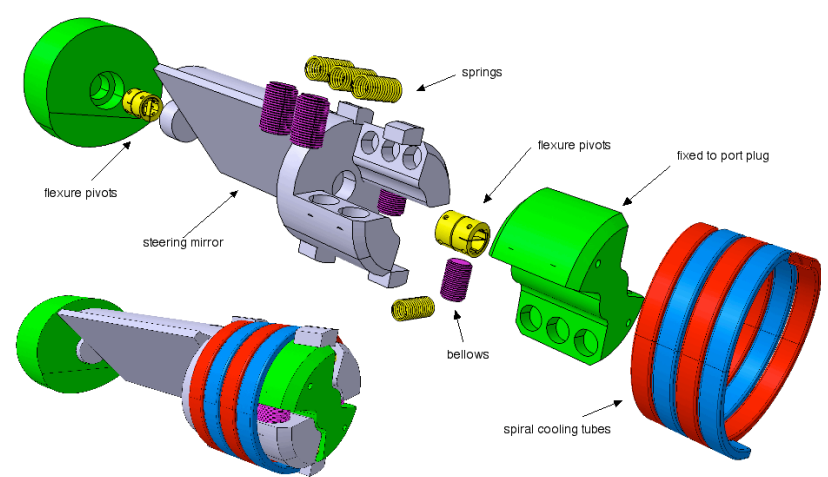

Figure 3. Illustration of the proposed frictionless and backlash-free steering mechanism to be used in the FS launcher. [7]

\section{NTM STABILISATION EFFICIENCY}


The achieved free space beam spot size varies from $20 \mathrm{~mm}$ to $\sim 44 \mathrm{~mm}$ along the resonance surface as shown in the figure 1 , to be contrasted with $\sim 56 \mathrm{~mm}$ to $\sim 115 \mathrm{~mm}$ for the RS launcher (averaged between the two rows). The resulting deposition location and driven current profile has been calculated using the ECWGB code [12] for the three ITER equilibria $\left(1_{\mathrm{i}} / \beta_{\mathrm{p}}\right)$ : $\operatorname{EoB} 2(0.7 / 0.65), 3 \mathrm{a}(0.7,1.0)$ and $5(1.0,0.8)$. Typically, the FS launcher can access the range $0.64<\rho_{\text {dep }}<$ 0.93 , which includes all of the $\mathrm{q}=2$ and $3 / 2$ surfaces for the investigated equilibria.

Of particular interest is a narrow current density profile $\left(\mathrm{j}_{\mathrm{CD}}\right)$ needed for stabilizing the Neoclassical tearing Mode (NTM). The narrow beam width provided by the FS launcher results in a more peaked current density profile achieving above marginal performance $\left(\eta_{\mathrm{NTM}}>1.2\right)$ on all the $\mathrm{q}=2$ and $3 / 2$ surfaces of the calculated equilibria, see table 1 , with $1.82 \leq \mathrm{j}_{\mathrm{CD}} / \mathrm{j}_{\mathrm{BS}} \leq 3.54$ and a total injected power of $20 \mathrm{MW}$ (assumes a transmission efficiency of $83 \%$ from gyrotron to plasma). The FS launcher provides on average a factor of 3 increase in $\mathrm{j}_{\mathrm{CD}}$ relative to the $\mathrm{RS}$ launcher for the $\mathrm{q}=2$ flux surfaces and a factor of 4.3 for the $q=3 / 2$ for all equilibria studied. This implies that $6.7 \mathrm{MW}(4.7 \mathrm{MW})$ in a FS launcher system is equivalent to $20 \mathrm{MW}$ in a RS launcher system to obtain an average performance for stabilizing the $q=2(q=3 / 2)$ NTM.

TABLE 1. Comparison of the NTM stabilization efficiency $\left(\mathrm{j}_{\mathrm{CD}} / \mathrm{j}_{\mathrm{BS}}\right)$ for the relevant rational surfaces and equilibria.

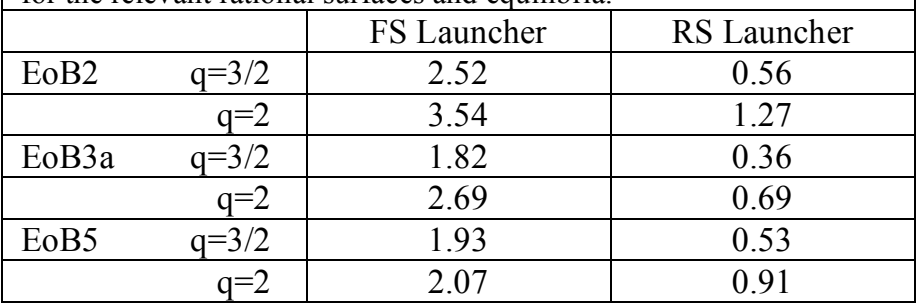

\section{EXTENDED PHysiCs ApPliCATIONS}

The rotation of the steering mirror can be increased so that the $\mathrm{j}_{\mathrm{CD}}$ deposition extends beyond the range necessary for NTM stabilization. At present the equatorial port launcher cannot effectively access the region beyond $\rho \sim 0.4$, while the upper port launcher does not extend inside $\rho \sim 0.64$ [13]. Increasing the FS scanning range downward bridges the gap allowing application of ECCD to all flux surfaces inside $\rho<0.9$. This could potentially offer control of the sawtooth instability [14] $\left(\mathrm{j}_{\mathrm{CD}}\right.$ deposition near the $\mathrm{q}=1$ surface) or the Frequently Interrupted Regime (FIR) [15] for all potential equilibria. Alternatively, the deposition of the FS launcher can be extended outward for potential de-stabilisation of the Edge Localised Mode (ELM). The ELM frequency has been increased by driving current locally in the plasma edge [16], which could be obtained by applying $j_{C D}$ at $\rho>0.95$. Preliminary ray tracing calculations using TORAY-GA [17, 18] have demonstrated that current can be driven over the range of $0.3 \leq \rho \leq 0.98$, see figure 4 . Even though the launch position is relatively high and the path length through the plasma is long, the absorption profile remains narrow since the resonance surface is nearly tangential to the flux surfaces. Dedicated design and ray tracing calculations are in progress for further optimization of the extended performance launcher design.

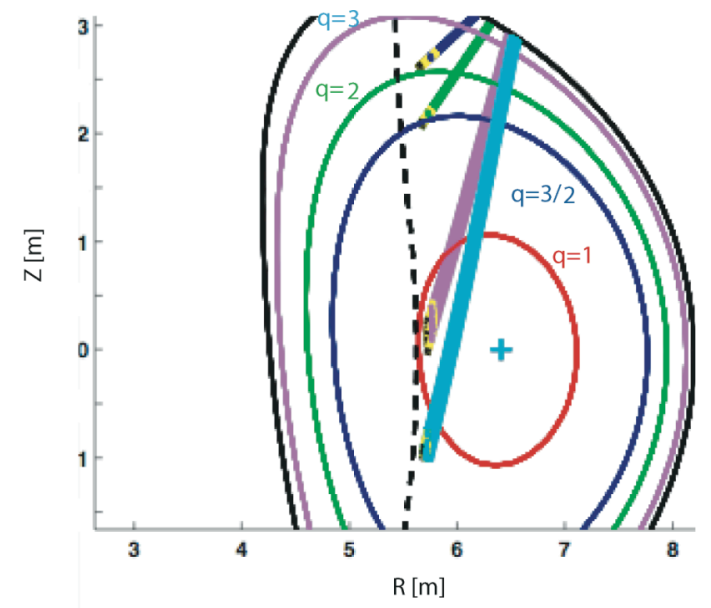

Figure 4. Ray tracing calculations using TORAY-GA for the extended performance FS launcher. [7]

\section{CONCLUSSIONS}

- The upper port launcher is being designed with the goals of optimum flexibility and reliability. Thus two possible launcher designs (RS and FS) are being investigated in parallel for the ITER upper port. The FS launcher provides a factor of 3 (4.3) increase in stabilizing the $q=2(3 / 2)$ NTM over that offered by the RS launcher. The steering range of the FS launcher can be increased to provide overlap with the Equatorial port launcher and permit extended physics applications beyond NTM stabilization, such as sawtooth, FIR and ELM control.

\section{ACKNOWLEDGMENT}

This work was supported in part by the Swiss National Science Foundation and the European Fusion Development Agreement.

\section{REFERENCES}

[1] H. Zohm et al, "Comparison of the performance of different options for the ITER ECRH Upper Laucnher", Accepted for publication JCPS IAEA-TM on ITER/ECRH (2005).

[2] C.P. Moeller, "A method of remotely steering a microwave beam launched from a highly overmoded corrugated waveguide", Proc. 23rd Int. Conf. on IRMMW, 1998.

[3] A.G.A. Verhoeven et al. "The remote-steering ECRH upper launcher for ITER", Accepted for publication JCPS IAEA-TM on ITER/ECRH (2005).

[4] D. Rondon et al, "Integration of a dog-leg beam routing for the remote steering upper port launcher for ITER" Accepted for publication JCPS IAEA-TM on ITER/ECRH (2005). 
[5] Henderson, M. A., Chavan, R., and Sanchez, F., "FS Launcher Study", Lausanne Research Report, LRP 791/04

[6] Chavan, R., Henderson, M. A., and Sanchez, F., "An Alternative ECRH Front Steering Launcher for the ITER Upper Port", submitted to Fusion Engineering and Design.

[7] Henderson et al, "The Front Steering Launcher Design for the ITER ECRH Upper Port", Accepted for publication JCPS IAEA-TM on ITER/ECRH (2005).

[8] Takahashi, K. et al, FED, 66 (2003) 473.

[9] Takahashi et al, "Development of Equatorial EC Launcher Components for ITER", Accepted for publication JCPS IAEA-TM on ITER/ECRH (2005).

[10] G. Ramponi, D.Farina and S. Nowak, "Capabilities of the ITER ECRH Upper Launcher at low magnetic fields", Accepted for publication JCPS IAEA-TM on ITER/ECRH (2005).
[11] Chavan, M. Henderson and F. Sanchez, "A Frictionless Steering Mechanism for the Front Steering ECCD ITER Upper Port Launcher", Accepted for publication JCPS IAEA-TM on ITER/ECRH (2005).

[12] Farina, D. et al, "ECWGB: a beam tracing code for EC heating and current drive", Report FP 03/06 (October 2003), http://www.ifp.cnr.it/

[13] F. Volpe, "Resiliency of ITER ECRH Upper Launcher to Steering Errors and Changes of Profiles and Integration with Equatorial Launcher"Accepted for publication JCPS IAEA-TM on ITER/ECRH (2005).

[14] Angioni, C. et al, Nucl. Fusion 43 (2003) 455-468.

[15] Gunter, S. et al, Nucl. Fusion 44 (2004) 524-532.

[16] Nave, M.F. et al, Nucl. Fusion 39 (1999) 1567.

[17] Matsuda, K., IEEE Trans. Plasma Sci., PS-17, 6 (1989).

[18] Cohen, R.H., Phys. Fluids 44, 139 (2002). 